\title{
Coronal loop density profile estimated by forward modelling of EUV intensity
}

\author{
D. J. Pascoe, C. R. Goddard, S. Anfinogentov, and V. M. Nakariakov
}

\author{
Centre for Fusion, Space and Astrophysics, Department of Physics, University of Warwick, CV4 7AL, UK \\ e-mail: D. J.Pascoe@warwick.ac.uk
}

Received 18 January 2017 / Accepted 5 March 2017

\begin{abstract}
Aims. The transverse density structuring of coronal loops was recently calculated for the first time using the general damping profile for kink oscillations. This seismological method assumes a density profile with a linear transition region. We consider to what extent this density profile accounts for the observed intensity profile of the loop, and how the transverse intensity profile may be used to complement the seismological technique.

Methods. We use isothermal and optically transparent approximations for which the intensity of extreme ultraviolet (EUV) emission is proportional to the square of the plasma density integrated along the line of sight. We consider four different models for the transverse density profile; the generalised Epstein profile, the step function, the linear transition region profile, and a Gaussian profile. The effect of the point spread function is included and Bayesian analysis is used for comparison of the models.

Results. The two profiles with finite transitions are found to be preferable to the step function profile, which supports the interpretation of kink mode damping as being due to mode coupling. The estimate of the transition layer width using forward modelling is consistent with the seismological estimate.

Conclusions. For wide loops, that is those observed with sufficiently high spatial resolution, this method can provide an independent estimate of density profile parameters for comparison with seismological estimates. In the ill-posed case of only one of the Gaussian or exponential damping regimes being observed, it may provide additional information to allow a seismological inversion to be performed. Alternatively, it may be used to obtain structuring information for loops that do not oscillate.
\end{abstract}

Key words. magnetohydrodynamics - Sun: atmosphere - Sun: corona - Sun: magnetic fields - Sun: oscillations - waves

\section{Introduction}

Large amplitude standing kink oscillations of coronal loops were first observed using the Transition Region And Coronal Explorer (TRACE; Aschwanden et al. 1999, 2002; Nakariakov et al. 1999). The Atmospheric Imaging Assembly (AIA; Lemen et al. 2012; Boerner et al. 2012) onboard the Solar Dynamics Observatory (SDO) has greatly increased their detection (e.g. Zimovets \& Nakariakov 2015; Goddard et al. 2016) and also led to the discovery of the low amplitude decayless regime of standing kink oscillations (Nisticò et al. 2013; Anfinogentov et al. 2013, 2015). The strong damping of propagating kink waves and large amplitude standing kink waves is attributed to mode coupling or resonant absorption (e.g. reviews by Pascoe 2014; De Moortel et al. 2016, which we can also expect to apply to low amplitude decayless oscillations if the damping is compensated by some persistent driving mechanism (e.g. Nakariakov et al. 2016). The presence of a smooth transition from the interior of an overdense coronal loop to the surrounding environment provides a continuous range of local Alfvén speeds. This condition allows mode coupling to occur, which results in the damping of kink oscillations as energy is transferred to Alfvén waves in the transition layer. The damping envelope of the kink oscillation is a continuous function (Hood et al. 2013) but may be approximated by a piecewise general damping profile (Pascoe et al. 2013; Arregui et al. 2013) consisting of a Gaussian stage (Pascoe et al. 2012, 2015) followed by an exponential stage (e.g. Ruderman \& Roberts 2002; Goossens et al. 2002). Direct observational evidence for the Gaussian damping regime
(De Moortel et al. 2002; Pascoe et al. 2016c), the seismological application of the general damping profile (Pascoe et al. 2016b, 2017), and the damping rates of spatially-resolved longitudinal harmonics (Pascoe et al. 2016a) support the interpretation of standing kink oscillations being damped by mode coupling.

The relationship between the density profile of a coronal loop and its appearance in EUV images such as those produced by SDO/AIA is complicated and has motivated numerous studies (e.g. De Moortel \& Bradshaw 2008; Owen et al. 2009; Taroyan \& Bradshaw 2014; Yuan \& Van Doorsselaere 2016). The emission by plasma at a particular EUV wavelength depends on the density and temperature and may include contributions from a large number of spectral lines. Coronal plasma is optically thin and so multiple structures or waves along the observational line of sight (LOS) appear superposed (e.g. De Moortel \& Pascoe 2012). This may also include an oscillating loop having a time-dependent superposition with itself (Cooper et al. 2003a). The measured intensity also depends on the properties of the particular instrument (e.g. Poduval et al. 2013). In this letter we consider a simple forward modelling procedure that allows us to estimate the loop density profile. Our approach, described in Sect. 2, is similar to Aschwanden et al. (2003) and Aschwanden \& Nightingale (2005) who modelled TRACE $171 \AA$ data using a density profile with a sinusoidal transition layer, and Aschwanden et al. (2007) who considered different loop density profiles. We estimate the spatial scale of the sub-structure of the coronal loop in the context of a monolithic loop, which may also correspond to the 
characteristic scale of multi-threaded coronal structures (e.g. Lenz et al. 1999; Aschwanden et al. 2000; Brooks et al. 2012; Antolin et al. 2015).

\section{Method}

We consider four different models for the transverse loop density profile. The step function profile (Model $S$ ) describes an overdense loop in terms of an internal density $\rho_{0}$, which is greater than the external density $\rho_{\mathrm{e}}$, and a minor radius $R$. Analytical solutions for the behaviour of magnetohydrodynamic waves in a cylindrical loop with this profile have been derived by Edwin \& Roberts (1983). We consider the transverse density profile $\rho(r)$ for a loop with a cylindrically symmetric crosssection and radial coordinate $r$ to be

$\rho(r)= \begin{cases}A, & |r| \leq R \\ 0, & |r|>R\end{cases}$

where $A=\rho_{0}-\rho_{\mathrm{e}}$ is the loop density enhancement.

The generalised symmetric Epstein profile (Model $E$ ) is defined as

$\rho(r)=A \operatorname{sech}^{2}\left(\frac{|r|}{R}\right)^{p}$,

which describes a smooth profile with a steepness determined by the parameter $p$. This profile has been used in many analytical (e.g. Nakariakov \& Roberts 1995; Cooper et al. 2003b; Macnamara \& Roberts 2011) and numerical studies (e.g. Nakariakov et al. 2005; Inglis et al. 2009; Pascoe \& De Moortel 2014; Pascoe \& Nakariakov 2016).

The linear transition layer profile (Model $L$ ) is given by

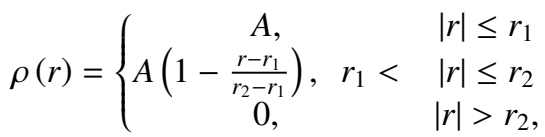

where $r_{1}=R(1-\epsilon / 2), r_{2}=R(1+\epsilon / 2)$, and $\epsilon=l / R$ is the transition layer width $l$ normalised to $R$ and defined to be in the range $\epsilon \in[0,2]$. The use of the linear transition layer profile in seismology is motivated by the availability of the full analytical solution for the damping envelope (Hood et al. 2013). However, the mechanism is insensitive to the details of fine structure (e.g. Terradas et al. 2008; Pascoe et al. 2011) and other profiles can be considered, such as a sinusoidal (e.g. Goossens et al. 2002; Roberts 2008) or parabolic (Arregui et al. 2015) transition layer profile.

We also consider a Gaussian density profile (Model $G$; e.g. Aschwanden et al. 2007) given by

$\rho(r)=A \exp \left(-\frac{r^{2}}{2 R^{2}}\right)$.

Examples of the four model profiles are given in Fig. 1, with the magnitude of all parameters $(A, R, p, \epsilon)$ taken to be unity.

The step function density profile is a limiting case of both the generalised Epstein $(p \rightarrow \infty)$ and linear transition layer $(\epsilon \rightarrow 0)$ profiles. Since it describes the case with no continuous transition between the higher density core and lower density background, it is also the only profile for which the damping of kink oscillations cannot be accounted for by mode coupling. We use the Bayesian evidence for Model $S$ to be a common normalisation for our test of Models $L$ and $E$ (see Bayes factor $K_{i S}$ in Table 1). We also compare our models to Model $G$ (using the Bayes factor $K_{i G}$ )

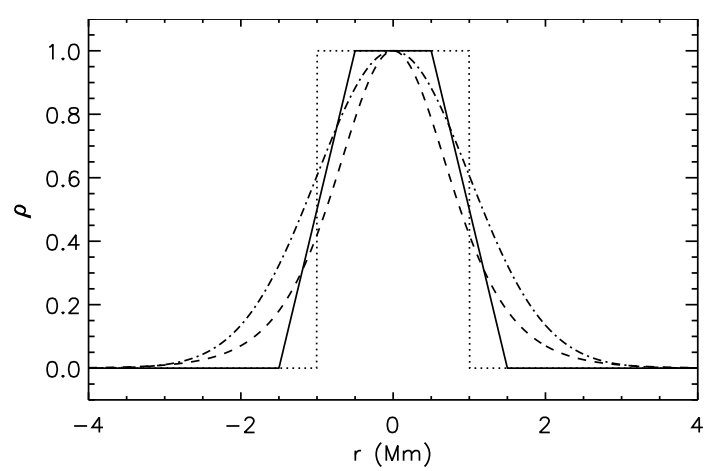

Fig. 1. Transverse loop density profiles used in our analysis; linear transition layer (Model $L$, solid line), Epstein profile (Model E, dashed line), step function (Model $S$, dotted line) and Gaussian (Model $G$, dash-dot line).

Table 1. Parameters for our density profile models $M_{i}$ for slit 10 .

\begin{tabular}{rrrrrrr}
\hline \hline$M_{i}$ & $A$ & $x_{0}(\mathrm{Mm})$ & $R(\mathrm{Mm})$ & $\epsilon, p$ & $K_{i S}$ & $K_{i G}$ \\
\hline$L$ & $0.72_{-0.04}^{+0.04}$ & $11.5_{-0.1}^{+0.1}$ & $4.51_{-0.13}^{+0.12}$ & $0.59_{-0.15}^{+0.14}$ & 20.6 & 46.3 \\
$E$ & $0.72_{-0.04}^{+0.04}$ & $11.5_{-0.1}^{+0.1}$ & $4.68_{-0.13}^{+0.13}$ & $3.85_{-0.94}^{+1.69}$ & 19.6 & 45.3 \\
$S$ & $0.66_{-0.04}^{+0.04}$ & $11.5_{-0.1}^{+0.1}$ & $4.47_{-0.15}^{+0.15}$ & - & - & 25.6 \\
$G$ & $1.06_{-0.11}^{+0.14}$ & $11.7_{-0.3}^{+0.3}$ & $3.68_{-0.45}^{+0.59}$ & - & -25.6 & - \\
\hline
\end{tabular}

which describes a continuously varying density profile, that is with no localisation of the transition layer.

Observations of hot coronal loops reveal them to be multithermal (e.g. Schmelz et al. 2010, 2014; Nisticò et al. 2014). However, Aschwanden \& Nightingale (2005) found the majority of cooler loops to be well-approximated as isothermal, and Warren et al. (2008) found isolated loops typically have very narrow temperature variation across the loop. Differential emission measure (DEM) analysis of our particular loop suggests a temperature in the range 0.75-1.25 MK (Pascoe et al. 2017). The use of the isothermal approximation allows the intensity profile to be calculated simply as the square of the density integrated along the LOS. For Model $S$, the integrated loop intensity could be readily calculated as $A^{2} d$, where the loop depth $d$ along the LOS is the chord length of the circular loop cross-section. However, since equivalent expressions are not available for the other models we instead calculate the loop intensity numerically by constructing a 2D (Cartesian) density profile for the radial profiles given in Eqs. (1)-(4) with

$r=\sqrt{\left(x-x_{0}\right)^{2}+\left(y-y_{0}\right)^{2}}$.

Here $x$ is the direction transverse to the loop, the loop centre is at $x_{0}$, and $y$ is the direction along the LOS. The values of $y$ and $y_{0}$ are arbitrary and so we take $y$ to have the same range as $x$ and $y_{0}=\langle y\rangle$. In addition to the contribution from the dense loop given by Eqs. (1)-(4), the density profile also contains a background contribution which is described by a second order polynomial in space. This corresponds to the emission from the background plasma (including any structures other than the loop). The intensity profile is smoothed to simulate the effect of the point spread function (PSF). We use a Gaussian kernel with $\sigma=1.019$ pixels, corresponding to the $171 \AA$ SDO/AIA channel (Grigis et al. 2013). The measured intensity profile consists of 44 data points, while our 2D density profile 


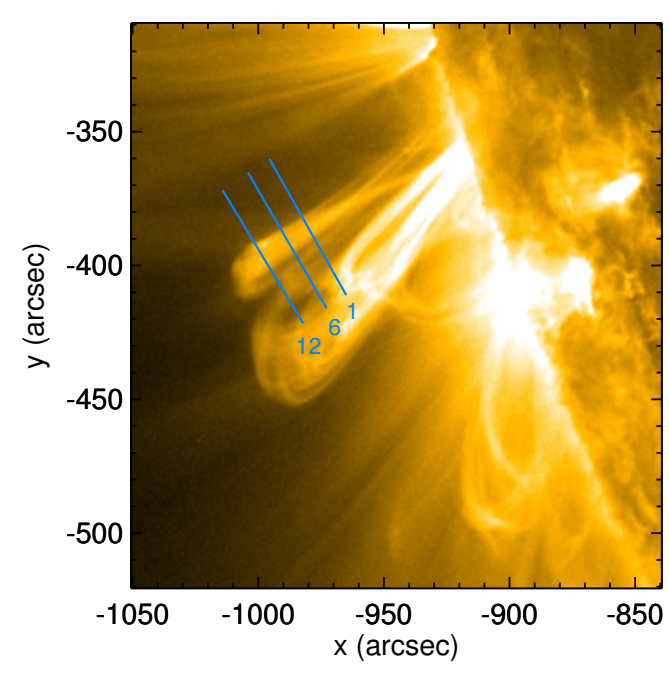

Fig. 2. SDO/AIA $171 \AA$ image of the analysed loop, observed 08:58:00 UT on 30 May 2012. The blue lines indicate the locations of the 12 slits used to generate transverse intensity profiles.

is calculated at a higher resolution of $440 \times 440$ pixels, that is a multiplication factor of 10 (convergence tests indicate consistent results for factors $\geq 7$ ). The model intensity profile is then interpolated onto the original transverse coordinates and compared with the observational data $D$ using the Bayesian inference and Markov chain Monte Carlo (MCMC) methods described in Pascoe et al. (2017). Calculating the Bayes factor $B_{i j}$ (Jeffreys 1961; Kass \& Raftery 1995) allows quantitative comparison of our four density profile models

$B_{i j}=\frac{P\left(D \mid M_{i}\right)}{P\left(D \mid M_{j}\right)}, \quad K_{i j}=2 \ln B_{i j}$.

Values of $K_{i j}$ greater than 2, 6, and 10 correspond to "positive", "strong", and "very strong" evidence for model $M_{i}$ over $M_{j}$, respectively, and negative values imply evidence for $M_{j}$ over $M_{i}$.

\section{Results}

We apply the method described in Sect. 2 to Loop \#3 from Pascoe et al. (2016b, 2017), chosen since it has the largest radius $(R \approx 4 \mathrm{Mm})$ of the four coronal loops analysed and so provides the greatest spatial information. Our method did not produce well-constrained values of $\epsilon$ for the three other loops, which have $R \lesssim 2 \mathrm{Mm}$, although instruments with higher spatial resolution than SDO/AIA would allow us to extend its applicability. This is also the loop for which the seismological estimate for $\epsilon$ is most narrowly constrained by its oscillation. The seismologically determined density profile parameters calculated in Pascoe et al. (2017) are $\rho_{0} / \rho_{\mathrm{e}}=2.96_{-0.66}^{+1.00}$ and $\epsilon=0.49_{-0.12}^{+0.23}$, where the parameter ranges correspond to the $95 \%$ credible intervals. The lower estimate for the density contrast in comparison with that in Pascoe et al. (2016b) results from taking the decayless component into account when analysing the kink oscillation. The loop is shown in Fig. 2 with the locations of 12 equally spaced slits used to generate transverse intensity profiles indicated by blue lines. The minor radius $R$ of the loop is observed to increase with height $z$ (measured along the loop axis). Consequently, slits taken higher up provide greater spatial information for the loop than those lower down. Higher slits may also benefit from fewer additional EUV sources along the LOS. On the other hand, we avoid slits too close to the apex of the loop $\left(z \sim r_{\mathrm{c}}-R \approx 70 \mathrm{Mm}\right.$, where $r_{\mathrm{c}}$ is the loop major radius) since here the intensity profile is complicated by the curved geometry.

Each intensity profile is analysed using the four models. Figure 3 and Table 1 summarise the results for one particular slit (slit 10). The left panel of Fig. 3 shows the observational intensity profile (symbols) and the profile for Model $L$ (blue line). The middle panel shows the histogram for $\epsilon$. The dotted and dashed lines denote the median value and the $95 \%$ credible interval, which also correspond to the values quoted in Table 1. The right panel shows the loop density profiles (using median values of sampled parameters) for each of the four models and indicates that models $E$ and $L$ produce very similar results.

Figure 4 shows the dependence of $\epsilon$ and $R$ with height for Model $L$. The loop is found to expand with height, while $\epsilon$ remains consistent with the seismological estimate (dotted lines). Generally, the value of $\epsilon$ obtained using forward modelling is less constrained than the seismological estimate. The right panel shows Bayes factors calculated for model comparison. For lower slits (with smaller $R$ ) there is no statistical evidence to prefer Model $L$ or $E$ over Model $S$, indicating the effects of LOS integration over a circular cross-section and the PSF are sufficient to account for the smoothness of the loop intensity profile. However, the evidence in favour of the two profiles with transition layers greatly increases over the step function for higher slits with effectively higher resolution, surpassing the requirements for "strong" $\left(K_{i j}>6\right)$ and "very strong" $\left(K_{i j}>10\right)$ evidence. For this data, there is no statistical evidence to distinguish between Models $L$ and $E\left(\left|K_{E L}\right| \lesssim 2\right)$, consistent with these models producing very similar results. Models $L, E$, and $S$ all have very strong evidence over Model $G$. For this loop we therefore find that a density model with a transition region ( $L$ or $E$ ) provides a better account of the intensity profile than a profile without a transition region $(S)$. On the other hand, the transition region is sufficiently localised that there is greater statistical evidence for Model $S$ than the fully inhomogeneous case of Model $G$.

\section{Discussion}

This study presents statistical evidence for the existence of a localised transition layer in the density profile of the coronal loop considered. Furthermore, the size of this layer is consistent for Models $L$ and $E$ (Fig. 3), and with the independent seismological estimate using a damped kink oscillation (Fig. 4).

Observations of kink oscillations for which only the Gaussian or exponential damping regime are detected produce illposed inversion problems since the damping time $\tau$ depends on both the density contrast and the layer width. Due to the effect of LOS integration, forward modelling of EUV intensity cannot reveal the density contrast of coronal loops since the intensity contrast also depends on an unknown column depth. However, we have demonstrated that the structure parameter $\epsilon$ may be estimated by forward modelling of the transverse intensity profile, and so combining this with the measured $\tau$ could allow the density contrast to be inferred. It may also be used to obtain structuring information for loops that do not oscillate, or to reveal any time-dependent variations in the cross-sectional profile (Aschwanden \& Schrijver 2011) which may be associated with non-linear effects (Goddard \& Nakariakov 2016).

Acknowledgements. This work is supported by the European Research Council under the SeismoSun Research Project No. 321141 (D.J.P., C.R.G., S.A., V.M.N.) and the STFC consolidated grant ST/L000733/1 (V.M.N.). The data is used courtesy of the SDO/AIA team. 

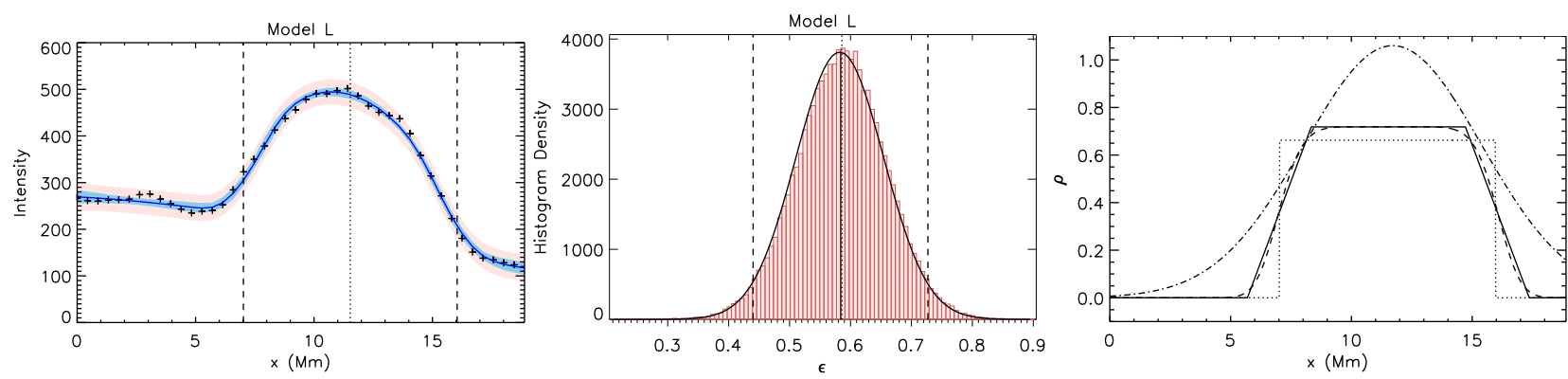

Fig. 3. SDO/AIA $171 \AA$ AUV intensity (left) across the loop is described by Model $L$ (blue line) which includes a background trend described by a second order polynomial. The shaded regions represent the $99 \%$ confidence intervals for the intensity predicted by the model, with (red) and without (blue) modelled noise. The vertical dotted and dashed lines denote $x_{0}$ and $x_{0} \pm R$, respectively. The middle panel shows the histogram of normalised layer width $\epsilon$ based on $10^{5}$ samples. The vertical dotted and dashed lines denote the median value and the $95 \%$ credible interval, respectively. Loop density profiles (right) for Models $L$ (solid), $E$ (dashed), $S$ (dotted), and $G$ (dash-dot).
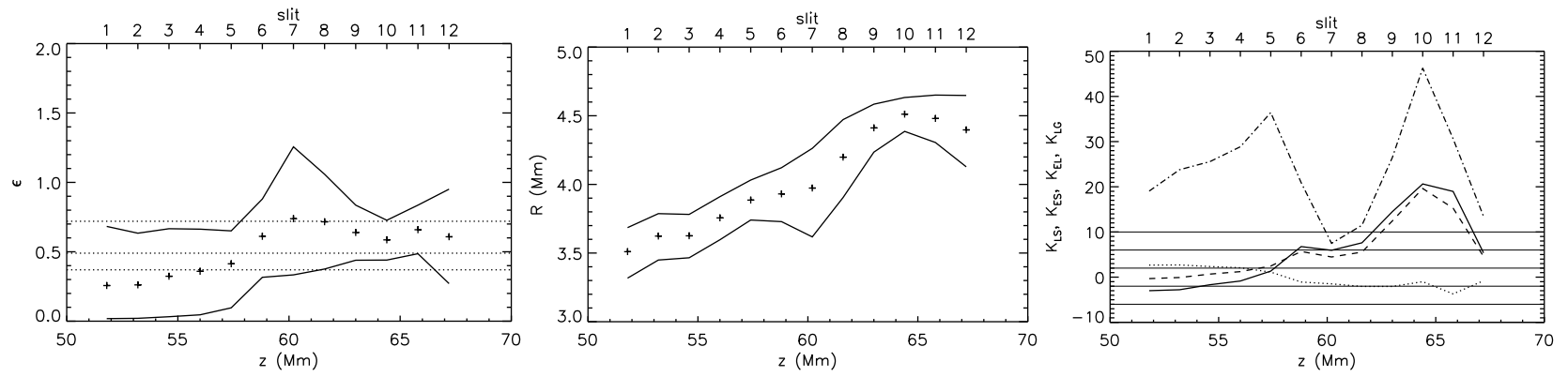

Fig. 4. Normalised layer width $\epsilon$ (left) and loop radius $R$ (middle) estimated by forward modelling. The symbols show the median values while the solid curves denote the $95 \%$ credible interval. The horizontal dotted lines correspond to the seismologically estimated values. The right panel shows the Bayes factors $K_{L S}$ (solid), $K_{E S}$ (dashed), $K_{E L}$ (dotted), and $K_{L G}$ (dash-dot).

\section{References}

Anfinogentov, S., Nisticò, G., \& Nakariakov, V. M. 2013, A\&A, 560, A107 Anfinogentov, S. A., Nakariakov, V. M., \& Nisticò, G. 2015, A\&A, 583, A136 Antolin, P., Vissers, G., Pereira, T. M. D., Rouppe van der Voort, L., \& Scullion, E. 2015, ApJ, 806, 81

Arregui, I., Asensio Ramos, A., \& Pascoe, D. J. 2013, ApJ, 769, L34

Arregui, I., Soler, R., \& Asensio Ramos, A. 2015, ApJ, 811, 104

Aschwanden, M. J., \& Nightingale, R. W. 2005, ApJ, 633, 499

Aschwanden, M. J., \& Schrijver, C. J. 2011, ApJ, 736, 102

Aschwanden, M. J., Fletcher, L., Schrijver, C. J., \& Alexander, D. 1999, ApJ, 520,880

Aschwanden, M. J., Nightingale, R. W., \& Alexander, D. 2000, ApJ, 541, 1059

Aschwanden, M. J., de Pontieu, B., Schrijver, C. J., \& Title, A. M. 2002, Sol. Phys., 206, 99

Aschwanden, M. J., Nightingale, R. W., Andries, J., Goossens, M., \& Van Doorsselaere, T. 2003, ApJ, 598, 1375

Aschwanden, M. J., Nightingale, R. W., \& Boerner, P. 2007, ApJ, 656, 577

Boerner, P., Edwards, C., Lemen, J., et al. 2012, Sol. Phys., 275, 41

Brooks, D. H., Warren, H. P., \& Ugarte-Urra, I. 2012, ApJ, 755, L33

Cooper, F. C., Nakariakov, V. M., \& Tsiklauri, D. 2003a, A\&A, 397, 765

Cooper, F. C., Nakariakov, V. M., \& Williams, D. R. 2003b, A\&A, 409, 325

De Moortel, I., Hood, A. W., \& Ireland, J. 2002, A\&A, 381, 311

De Moortel, I., \& Bradshaw, S. J. 2008, Sol. Phys., 252, 101

De Moortel, I., \& Pascoe, D. J. 2012, ApJ, 746, 31

De Moortel, I., Pascoe, D. J., Wright, A. N., \& Hood, A. W. 2016, Plasma Physics and Controlled Fusion, 58, 014001

Edwin, P. M., \& Roberts, B. 1983, Sol. Phys., 88, 179

Goddard, C. R., \& Nakariakov, V. M. 2016, A\&A, 590, L5

Goddard, C. R., Nisticò, G., Nakariakov, V. M., \& Zimovets, I. V. 2016, A\&A, 585, A 137

Goossens, M., Andries, J., \& Aschwanden, M. J. 2002, A\&A, 394, L39

Grigis, P., Yingna, S., \& Weber, M. 2013, Tech. Rep., AIA team

Hood, A. W., Ruderman, M., Pascoe, D. J., et al. 2013, A\&A, 551, A39

Inglis, A. R., van Doorsselaere, T., Brady, C. S., \& Nakariakov, V. M. 2009, A\&A, 503, 569

Jeffreys, H. 1961, Theory of Probability, 3rd edn. (Oxford)

Kass, R. E., \& Raftery, A. E. 1995, J. Am. Stat. Assoc., 90, 773

Lemen, J. R., Title, A. M., Akin, D. J., et al. 2012, Sol. Phys., 275, 17

Lenz, D. D., Deluca, E. E., Golub, L., et al. 1999, Sol. Phys., 190, 131
Macnamara, C. K., \& Roberts, B. 2011, A\&A, 526, A75

Nakariakov, V. M., \& Roberts, B. 1995, Sol. Phys., 159, 399

Nakariakov, V. M., Ofman, L., Deluca, E. E., Roberts, B., \& Davila, J. M. 1999, Science, 285, 862

Nakariakov, V. M., Pascoe, D. J., \& Arber, T. D. 2005, Space Sci. Rev., 121, 115

Nakariakov, V. M., Anfinogentov, S. A., Nisticò, G., \& Lee, D.-H. 2016, A\&A, 591, L5

Nisticò, G., Nakariakov, V. M., \& Verwichte, E. 2013, A\&A, 552, A57

Nisticò, G., Anfinogentov, S., \& Nakariakov, V. M. 2014, A\&A, 570, A84

Owen, N. R., De Moortel, I., \& Hood, A. W. 2009, A\&A, 494, 339

Pascoe, D. J. 2014, RA\&A, 14, 805

Pascoe, D. J., \& De Moortel, I. 2014, ApJ, 784, 101

Pascoe, D. J., \& Nakariakov, V. M. 2016, A\&A, 593, A52

Pascoe, D. J., Wright, A. N., \& De Moortel, I. 2011, ApJ, 731, 73

Pascoe, D. J., Hood, A. W., de Moortel, I., \& Wright, A. N. 2012, A\&A, 539, A37

Pascoe, D. J., Hood, A. W., De Moortel, I., \& Wright, A. N. 2013, A\&A, 551, A40

Pascoe, D. J., Wright, A. N., De Moortel, I., \& Hood, A. W. 2015, A\&A, 578, A99

Pascoe, D. J., Goddard, C. R., \& Nakariakov, V. M. 2016a, A\&A, 593, A53

Pascoe, D. J., Goddard, C. R., Nisticò, G., Anfinogentov, S., \& Nakariakov, V. M. 2016b, A\&A, 589, A136

Pascoe, D. J., Goddard, C. R., Nisticò, G., Anfinogentov, S., \& Nakariakov, V. M. 2016c, A\&A, 585, L6

Pascoe, D. J., Anfinogentov, S., Nisticò, G., Goddard, C. R., \& Nakariakov, V. M. 2017, A\&A, in press DOI: 10.1051/0004-6361/201629702

Poduval, B., DeForest, C. E., Schmelz, J. T., \& Pathak, S. 2013, ApJ, 765, 144

Roberts, B. 2008, in IAU Symp., Vol. 247, eds. R. Erdélyi, \& C. A. MendozaBriceno, 3

Ruderman, M. S., \& Roberts, B. 2002, ApJ, 577, 475

Schmelz, J. T., Saar, S. H., Nasraoui, K., et al. 2010, ApJ, 723, 1180

Schmelz, J. T., Pathak, S., Brooks, D. H., Christian, G. M., \& Dhaliwal, R. S. 2014, ApJ, 795, 171

Taroyan, Y., \& Bradshaw, S. J. 2014, Sol. Phys., 289, 1959

Terradas, J., Arregui, I., Oliver, R., et al. 2008, ApJ, 679, 1611

Warren, H. P., Ugarte-Urra, I., Doschek, G. A., Brooks, D. H., \& Williams, D. R. 2008, ApJ, 686, L131

Yuan, D., \& Van Doorsselaere, T. 2016, ApJS, 223, 24

Zimovets, I. V., \& Nakariakov, V. M. 2015, A\&A, 577, A4 